\title{
Atomap - Automated Analysis of Atomic Resolution STEM Images
}

\author{
Magnus Nord ${ }^{1,2}$, Per Erik Vullum ${ }^{1,3}$, Ian MacLaren ${ }^{2}$, Thomas Tybell ${ }^{4}$, Randi Holmestad $^{1}$ \\ 1. Dept. of Physics, Norwegian University of Science and Technology, NTNU, Trondheim, Norway \\ 2. SUPA, School of Physics and Astronomy, University of Glasgow, Glasgow, United Kingdom \\ 3. Materials and Chemistry, SINTEF, Trondheim, Norway \\ 4. Dept. of Electronic Systems, Norwegian University of Science and Technology, NTNU, Trondheim, \\ Norway
}

Atomic resolution scanning transmission electron microscopy (STEM) data contain a large amount of information about the structure of a crystalline material. Often, this information is hard to extract, due to the large number of atomic columns and large differences in intensity from sublattices consisting of different elements. Atomap is a semi-automatic, free and open source software tool for analysing both the position and shapes of atomic columns in STEM-images, using 2-D elliptical Gaussian distributions. In this work, the Atomap software is used on variants of the perovskite oxide structure, obtaining quantitative information from projected sublattices. Changes in the lattice parameters and shape of Acation columns from annular dark field images of perovskite oxide heterostructures are obtained. Furthermore, we demonstrate the functionality of Atomap by quantifying deviation from centrosymmerty of light atomic oxygen columns.

STEM is a well-established experimental technique for imaging the structure and composition of materials. With the advent of spherical aberration correctors, acquisition of atomic resolution STEM images has become routine. These images contain a wealth of information, such as intensity, shape and position of the atomic columns. These can be used to determine the local atomic structure of materials, which is usually not discernible using X-ray techniques. However, much of this detailed information is typically not utilised, due to a lack of practical and easily available methods for the analysis of this type of data. Atomap - a semi-automatic, free and open source software for analysing atomic resolution STEM data [1] - overcomes this problem. Example uses of Atomap are finding octahedral tilt patterns in perovskite oxides, ellipticity of atomic columns (which may reveal non-straight atomic columns along the beam direction), and deviation from centrosymmetry of the local structure.

The software is implemented in Python 3, and is available at http://atomap.org. Atomap relies heavily on the modelling functionality from HyperSpy [2], and works by firstly finding the most intense atomic columns in an image. The position of these atomic columns are refined using centre of mass. Next, each atomic column is fitted with a 2-D Gaussian, resulting in more precise determination of the position and the ability to "subtract" the intensity from the most intense atomic columns. The second most intense atomic columns can then be located, and the process is repeated for this sublattice, and any subsequent sublattices. The average 2-D atomic arrangement is found for each sublattice using the atomic positions, and the columns are automatically grouped into different atomic planes. This approach allows the effective measurement of the atomic positions of light elements, and for perovskite oxides this hence allows the oxygen atomic columns to be accurately fitted.

We used Atomap to study minute structural changes in an epitaxial perovskite oxide heterostructure. Datasets from a cross section sample of $\mathrm{La}_{0.7} \mathrm{Sr}_{0.3} \mathrm{MnO}_{3}(4 \mathrm{~nm}) / \mathrm{LaFeO}_{3}(4 \mathrm{~nm}) / \mathrm{SrTiO}_{3}-(111)$ (LSMO/LFO/STO) heterostructure [3] were acquired on a JEOL ARM200CF equipped with probe 
aberration corrector. While the software was tested on a perovskite oxide heterostructure projected along a $\langle 110\rangle$ direction, we note that it should work on any atomic resolution STEM data as long as the atomic columns are clearly resolved. Having found all the atomic positions, parameters such as the distance between atomic columns, deviation from centrosymmetric positions (Fig 1) and atom column ellipticity (Fig 2) can be mapped. We found that the shapes of the projected La/Sr columns vary over the same heterostructure: there is a clear elongation in the LFO layer and the ellipticity on this column correlates with the tilting of the oxygen octahedra. These data show that both oxygen and A-cations have shifted positions in the middle layer [4].

\section{References:}

[1] M. Nord et al., Advanced Structural and Chemical Imaging 3:9 (2017)

[2] http://hyperspy.org/

[3] I. Hallsteinsen et al., Physical Review B 94 (2016), p 201115

[4] The STEM work was carried out on the NORTEM infrastructure at the TEM Gemini Centre, NTNU, Norway. Images taken from [1] under the Creative Commons Attribution 4.0 International License.
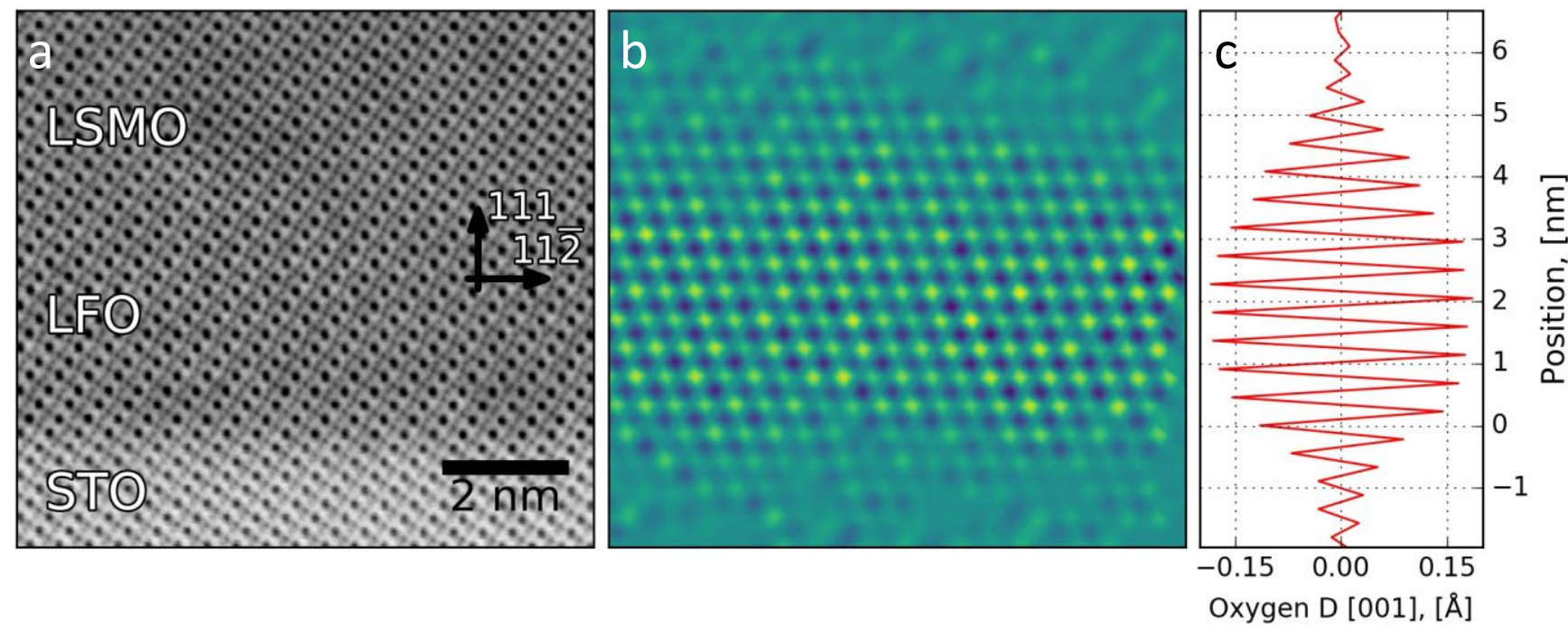

Figure 1. (a) Annular bright field image of LSMO/LFO/STO-(111) heterostructure. (b) Deviation from centro-symmetric position for oxygen columns in the $\langle 100\rangle$ direction. (c) sum of the deviation in (b) as a function of distance from the LFO/STO-interface.
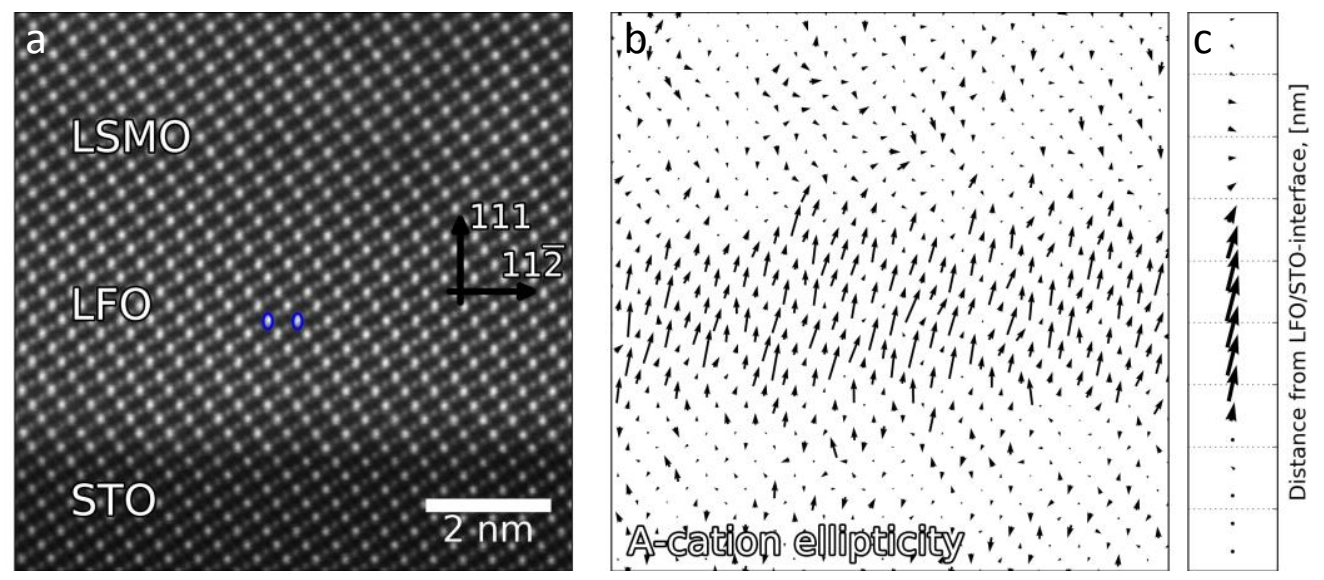

Figure 2. Annular dark field image of LSMO/ LFO/STO-(111) heterostructure. (b) Magnitude and direction of $\mathrm{La} / \mathrm{Sr}$ column ellipticity. (c) Sum of (b) as a function of distance from the LFO/STO-interface. 